\title{
Multi agent system architecture oriented Prometheus methodology design to facilitate security of cloud data storage
}

\begin{abstract}
Security plays an important role in the development of Multi Agent Systems (MAS). However, a careful analysis of software development processes shows that the definition of security requirements is, usually, considered after the design of the system. This is, mainly, due to the fact that agent oriented software engineering methodologies have not integrated security concerns throughout their developing stages. Designing a team of agents that can work together toward a common goal is one of the challenges in the research area of agentoriented software engineering. Prometheus is an agent-oriented software engineering methodology. The Prometheus Design Tool (PDT) is a graphical editor which supports the design tasks specified within the Prometheus methodology for designing agent systems. The tool propagates information where possible and ensures consistency between various parts of the design. The main purpose of this paper is to design MAS architecture that can be used to facilitate confidentiality, correctness assurance, availability and integrity of Cloud Data Storage (CDS) or cloud data center. The proposed MAS architecture includes five types of agents: Cloud Service Provider Agent (CSPA), Cloud Data Confidentiality Agent (CDConA), Cloud Data Correctness Agent (CDCorA), Cloud Data Availability Agent (CDAA) and Cloud Data Integrity Agent (CDIA).
\end{abstract}

Keyword: Agent-oriented methodology; Cloud computing; Cloud data storage; Multi agent system; Prometheus design tool; Prometheus methodology; Security 